\title{
Localization of coherently distributed source using a successive propagator method
}

\author{
Qianlin Cheng ${ }^{1, a}$, Donglin Yang ${ }^{1}$, Weitao Liu $^{1}$, You Sun ${ }^{1}$ and Xiaofei Zhang ${ }^{1,2,3}$ \\ ${ }^{1}$ College of Electronic and Information Engineering, Nanjing University of Aeronautics and \\ Astronautics, Nanjing, 210016, P.R.China \\ ${ }^{2}$ State Key Laboratory of Millimeter Waves, Southeast University, Nanjing, China, 210096. \\ ${ }^{3}$ National Mobile Communications Research Laboratory, Southeast University, Nanjing, China, \\ 210096.
}

achengqianlin_nuaa@163.com

Keywords: direction of arrival, coherently distributed source, successive PM algorithm.

\begin{abstract}
In this letter, the localization of coherently distributed (CD) is considered. With regard to the direction finding of $\mathrm{CD}$ source, the conventional method distributed source parameter estimation (DSPE) can provide stable estimation performance, while it also requires a complex two-dimensional spectral peak search for both nominal direction-of-arrivals (DOAs) and angular spreads. Thus, with rank reduction criterion, we derived a successive propagator method (S-PM) which exploits three decoupled one-dimensional search to replace an extensive one-dimension search to reduce the heavy cost. And the proposed algorithm can avoid the high-complexity eigen-decomposition procedure by using the linear operator-propagator. When compared to the convention ESPRIT-CD [8], S-PM has a better estimation performance with a low complexity. Several simulation results illustrate the effectiveness and improvements of the proposed algorithm.
\end{abstract}

\section{Introduction}

When it comes to the problem of direction-of-arrival (DOA) estimation, many works are generally based on a common hypothesis that the signals are generated from the point source. But when applied in some practical scenarios such as cellular mobile communication [1-3], low elevation radar target tracking, passive sonar systems [4] and radio propagation in tropospheric [5-6] etc., the effects of multipath will lead to a decrease in estimation performance. Hence, the distributed source model is introduced to better apply in the aforementioned scenes.

Many previous literatures have carried out research on the parameter estimation of one-dimensional coherently distributed (CD) source. In [7], a MUSIC-type algorithm called distributed source parameter estimation (DSPE) has superior estimation accuracy but suffers from the extensive two-dimensional search of nominal DOAs and angular spreads. The work in [8] proposed a low complexity algorithm with an unsatisfied estimation performance. And the work in [11] which also needs 2-D search is based on the OPM algorithm [14], which utilizes the propagator to obtain the noise subspace and estimates nominal DOAs and angular spreads through a similar spectrum search like DSPE-CD [7].

In this letter, a successive propagator method (S-PM) algorithm was proposed to further reduce the computational complexity. The estimated nominal DOAs and angular spreads can be easily obtained by searching three decoupled 1-D rather than 2-D DSPE-like spectrum. Thus, S-PM algorithm provides lower complexity when compared to the OPM algorithm in [11]. Moreover, the estimation performance of the proposed one is obviously superior to the ESPRIT-CD [8] algorithm. The remainder of this letter is organized as follows. Section 2 introduces the data model. The S-PM algorithm is proposed in section 3. In section 4, we give the performance analysis. Simulation results are presented in section 5 . Conclusion is summarized in section 6 . 


\section{Data model}

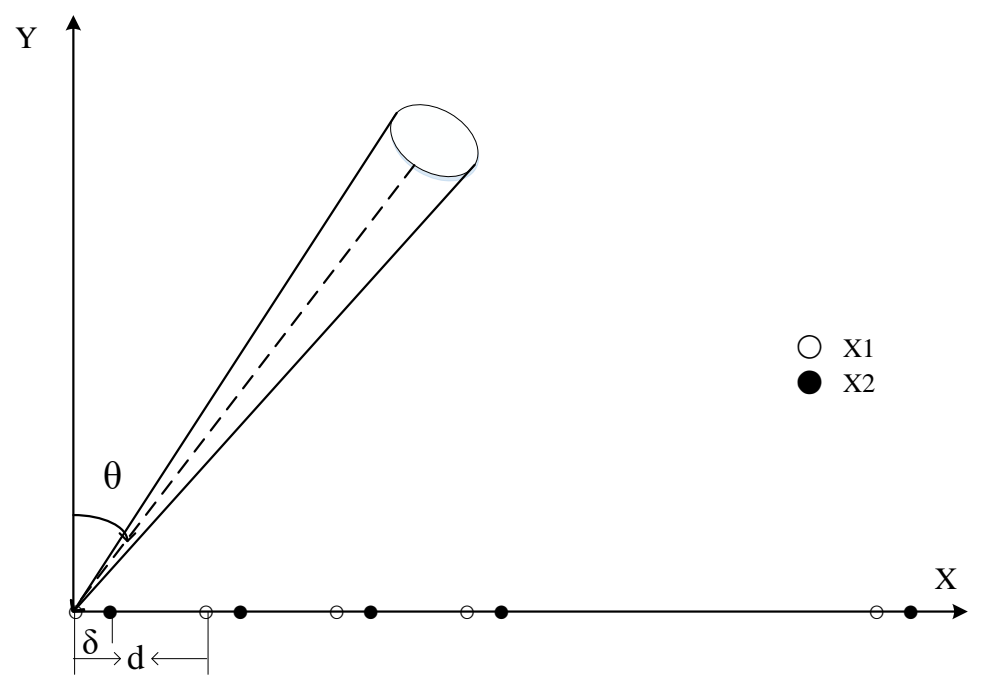

Fig.1 Array structure

Assume $K$ narrowband, far-field, uncorrelated CD sources impinging on the array shown in Fig.1. The array is composed of two identical linear subarrays which is consist of $M$ omnidirectional sensors with half-wavelength inter-element spacing, and $2 M \geq K$. And the constant displacement between two subarrays is $\delta$ and satisfies $\delta \ll \lambda / 2$. Hence, the received signal of the two subarrays $\mathrm{X}_{1}$ and $\mathrm{X}_{2}$ can be written in the form of continuous integral

$$
\begin{aligned}
& \mathbf{r}_{1}(t)=\sum_{i=1}^{k} \int_{-\pi / 2}^{\pi / 2} \mathbf{a}(\theta) s_{i}\left(\theta, t ; \boldsymbol{\Psi}_{i}\right) d \theta+\mathbf{n}_{1}(t), \\
& \mathbf{r}_{2}(t)=\sum_{i=1}^{k} \int_{-\pi / 2}^{\pi / 2} \mathbf{a}(\theta) s_{i}\left(\theta, t ; \boldsymbol{\Psi}_{i}\right) e^{-j 2 \pi \frac{\delta}{\lambda} \sin \theta} d \theta+\mathbf{n}_{2}(t),
\end{aligned}
$$

where $\mathbf{a}(\theta)$ denotes the $M \times 1$ response vector of the array at direction $\theta ; s_{i}\left(\theta, t ; \boldsymbol{\psi}_{i}\right)$ denotes the angular signal density of the $i$ th $\mathrm{CD}$ source; $\boldsymbol{\psi}_{i}=\left[\theta_{i}, \sigma_{i}\right]$ denotes the location parameter vector with nominal DOA $\theta_{i}$ and the angular spread $\sigma_{i}$ of the $i$ th CD source; $\mathbf{n}_{1}(t)$ and $\mathbf{n}_{2}(t)$ denote the complex-valued additive zero-mean white Gaussian noise vectors. $\lambda$ is the wavelength, and $t$ is the time index.

For CD source, the angular signal density $s_{i}\left(\theta, t ; \boldsymbol{\psi}_{i}\right)$ can be expressed as

(3)

$$
s_{i}\left(\theta, t ; \boldsymbol{\psi}_{i}\right)=s_{i}(t) \rho\left(\theta ; \boldsymbol{\psi}_{i}\right) \text {, }
$$

where $s_{i}(t)$ is the complex random signal of the ith source and $\rho\left(\theta ; \boldsymbol{\psi}_{i}\right)$ is the deterministic angular density function. Note that the index $i$ in $\rho\left(\theta ; \boldsymbol{\psi}_{i}\right)$ implies different sources may have different angular distribution.

Then we can define

$$
\begin{aligned}
& \mathbf{b}_{\mathbf{1}}\left(\boldsymbol{\Psi}_{i}\right)=\int_{-\pi / 2}^{\pi / 2} \mathbf{a}(\theta) \rho\left(\theta ; \boldsymbol{\psi}_{i}\right) d \theta, \\
& \mathbf{b}_{2}\left(\boldsymbol{\Psi}_{i}\right)=\int_{-\pi / 2}^{\pi / 2} \mathbf{a}(\theta) \rho\left(\theta ; \boldsymbol{\psi}_{i}\right) e^{-j 2 \pi \frac{\delta}{\lambda} \sin \theta} d \theta,
\end{aligned}
$$


where $\mathbf{b}_{1}\left(\boldsymbol{\psi}_{i}\right)$ and $\mathbf{b}_{2}\left(\boldsymbol{\psi}_{i}\right)$ are the generalized array manifold vectors of the two subarrays $\mathrm{X}_{1}$ and $\mathrm{X}_{2}$, respectively.

So the received signal of the array can be presented in the form of matrix as

$$
\mathbf{r}(t)=\left[\begin{array}{l}
\mathbf{r}_{1}(t) \\
\mathbf{r}_{2}(t)
\end{array}\right]=\left[\begin{array}{l}
\mathbf{B}_{1} \\
\mathbf{B}_{2}
\end{array}\right] \mathbf{s}(t)+\left[\begin{array}{l}
\mathbf{n}_{1}(t) \\
\mathbf{n}_{2}(t)
\end{array}\right],
$$

where $\quad \mathbf{B}_{1}=\left[\begin{array}{llll}\mathbf{b}_{1}\left(\psi_{1}\right) & \mathbf{b}_{1}\left(\psi_{2}\right), \cdots, \mathbf{b}_{1}\left(\psi_{k}\right)\end{array}\right] \quad, \quad \mathbf{B}_{2}=\left[\begin{array}{llll}\mathbf{b}_{2}\left(\psi_{1}\right) & \mathbf{b}_{2}\left(\psi_{2}\right), \cdots, \mathbf{b}_{2}\left(\psi_{k}\right)\end{array}\right] \quad$ and $\mathbf{s}(t)=\left[\begin{array}{llll}s_{1}(t) & s_{2}(t) & , \cdots, & s_{K}(t)\end{array}\right]^{T}$.

In the actual implementation of finite snapshots, the estimated covariance matrix $\hat{\mathbf{R}}$ can be denoted as $\hat{\mathbf{R}}=\frac{1}{L} \sum_{t=1}^{L} \mathbf{r}(t) \mathbf{r}^{H}(t)$, where $L$ is the number of snapshots.

\section{The proposed algorithm}

3.1 The preliminary estimation via $\mathbf{P M}$ algorithm. We can make a common assumption like many previous works [9-10] that the array manifold $\mathbf{B}$ is of full rank and the $K$ rows of $\mathbf{B}$ are linearly independent. Then the other $(2 M-K)$ rows of $\mathbf{B}$ can be expressed as a linear combination of the former $K$ rows. Assume that the first $\mathrm{K}$ rows of $\mathbf{B}$ are linearly independent. Then we partition $\mathbf{B}$ as

$$
\left.\mathbf{B}=\left[\begin{array}{l}
\mathbf{B}_{a} \\
\mathbf{B}_{b}
\end{array}\right]\right\} \begin{aligned}
& \} \text { rows } \\
& 2 M-K \text { rows }
\end{aligned}
$$

where the nonsingular $K \times K$ matrix $\mathbf{B}_{a}$ denotes the first $K$ rows of $\mathbf{B}$ and $\mathbf{B}_{b}$ denotes the last $(2 M-K)$ rows of $\mathbf{B}$.

And there exist a linear operator $\mathbf{P}$ between $\mathbf{B}_{a}$ and $\mathbf{B}_{b}$ that satisfies the following relationship

$$
\mathbf{B}_{b}=\mathbf{P B}_{a} \text {. }
$$

The propagator $\mathbf{P}$ can be estimated as [12]

$$
\mathbf{P}=\left(\hat{\mathbf{R}}_{1} \hat{\mathbf{R}}_{1}^{H}\right)^{-1} \hat{\mathbf{R}}_{1} \hat{\mathbf{R}}_{2}^{H},
$$

where $\hat{\mathbf{R}}_{1}$ and $\hat{\mathbf{R}}_{2}$ denote the first $K$ rows and the remaining rows of $\hat{\mathbf{R}}$ in (12)

Then define a matrix

$$
\mathbf{Q}=\left[\begin{array}{c}
\mathbf{I}_{K} \\
\mathbf{P}^{H}
\end{array}\right] \text {. }
$$

According to (14), 16), (17), it is easy to obtain that [13]

$$
\mathbf{Q B}_{a}=\left[\begin{array}{c}
\mathbf{I}_{K} \\
\mathbf{P}^{H}
\end{array}\right] \mathbf{B}_{a}=\mathbf{B} .
$$

Considering introducing $\mathbf{Q}$ into the projection operator of the noise subspace, we replace it with its orthogonalized matrix $\mathbf{U}=\mathbf{Q}\left(\mathbf{Q}^{H} \mathbf{Q}\right)^{-1 / 2}$ and we can also get

$\mathbf{U}^{H} \mathbf{B}=\mathbf{0}$ 
Then we can utilize $\mathbf{Q}_{a}$ and $\mathbf{Q}_{b}$ to denote the first $M-1$ rows and the last $M-1$ rows of $\mathbf{Q}$, respectively. Then we can get

$$
\left[\begin{array}{l}
\mathbf{Q}_{a} \\
\mathbf{Q}_{b}
\end{array}\right] \mathbf{B}_{a}=\left[\begin{array}{c}
\mathbf{B}_{c} \\
\mathbf{B}_{d}
\end{array}\right]=\left[\begin{array}{c}
\mathbf{B}_{a} \\
\mathbf{B}_{a} \boldsymbol{\Phi}_{r}
\end{array}\right],
$$

where $\mathbf{B}_{c}$ and $\mathbf{B}_{d}$ stand for the first $M-1$ rows and the last $M-1$ rows of $\mathbf{B}$. And there exist the following relationship $\mathbf{Q}_{a}^{+} \mathbf{Q}_{b}=\mathbf{B}_{a} \boldsymbol{\Phi}_{r} \mathbf{B}_{a}$.

Then the preliminary estimation of nominal DOAs $\hat{\boldsymbol{\theta}}_{i n i}=\left[\hat{\theta}_{i 1}, \hat{\theta}_{i 2}, \cdots, \hat{\theta}_{i k}\right]$ could be estimated by performing eigenvalue decomposition on $\boldsymbol{\Phi}_{r}$.

3.2 The spectral peak search. We utilize the initial nominal DOAs $\hat{\boldsymbol{\theta}}_{i n i}=\left[\hat{\theta}_{i 1}, \hat{\theta}_{i 2}, \cdots, \hat{\theta}_{i K}\right]$ to implement the first 1D spectral peak search. The spatial spectrum function is given by

$$
P_{1}\left(\hat{\theta}_{i i}, \sigma_{i i}\right)=\frac{1}{\left\|\mathbf{b}^{H}\left(\hat{\theta}_{i i}, \sigma_{i i}\right) \mathbf{U}\right\|^{2}} \quad i=1, \cdots, K,
$$

where $\mathbf{b}\left(\hat{\theta}_{i i}, \sigma_{i i}\right)=\left[\mathbf{b}_{1}\left(\hat{\theta}_{i i}, \sigma_{i i}\right), \mathbf{b}_{2}\left(\hat{\theta}_{i i}, \sigma_{i i}\right)\right]^{T}$ and $\hat{\theta}_{i i} \in \hat{\boldsymbol{\theta}}_{i n i}$.

By one-dimensional search via $\sigma_{i i}$, we can obtain the initial estimation of the angular spreads $\hat{\boldsymbol{\sigma}}_{i n i}=\left[\hat{\sigma}_{i 1}, \hat{\sigma}_{i 2}, \cdots, \hat{\sigma}_{i K}\right]$.

3.3 The second spectral peak search. In this section, we use the estimation of the angular spreads $\hat{\boldsymbol{\sigma}}_{i n i}=\left[\hat{\sigma}_{i 1}, \hat{\sigma}_{i 2}, \cdots, \hat{\sigma}_{i K}\right]$ in 3.2 to implement the second 1D search. Then the spatial spectrum function could be expressed as

$$
P_{2}\left(\theta_{i}, \hat{\sigma}_{i i}\right)=\frac{1}{\left\|\mathbf{b}^{H}\left(\theta_{i}, \hat{\sigma}_{i i}\right) \mathbf{U}\right\|^{2}} \quad i=1, \cdots, K,
$$

where $\mathbf{b}\left(\theta_{i}, \hat{\sigma}_{i i}\right)=\left[\mathbf{b}_{1}\left(\theta_{i}, \hat{\sigma}_{i i}\right), \mathbf{b}_{2}\left(\theta_{i}, \hat{\sigma}_{i i}\right)\right]^{T}$ and $\hat{\sigma}_{i i} \in \hat{\boldsymbol{\sigma}}_{i n i}$.

The second estimation of nominal DOAs $\hat{\boldsymbol{\theta}}_{\mathrm{sec}}=\left[\hat{\theta}_{\mathrm{s} 1}, \hat{\theta}_{\mathrm{s} 2}, \cdots, \hat{\theta}_{\mathrm{s} K}\right]$ can be obtained by 1D search via $\theta_{i}$. The simulation results in section 5 will demonstrate that it is more accurate than the initial estimators.

3.4 The third spectral peak search. Based on the aforementioned works, we can utilize the second estimation of nominal DOAs $\hat{\boldsymbol{\theta}}_{\mathrm{sec}}=\left[\hat{\theta}_{\mathrm{s} 1}, \hat{\theta}_{\mathrm{s} 2}, \cdots, \hat{\theta}_{\mathrm{sK}}\right]$ in 3.3 to implement the third 1D search. The spatial spectrum function can be presented as

$$
P_{3}\left(\hat{\theta}_{s i}, \sigma_{s i}\right)=\frac{1}{\left\|\mathbf{b}^{H}\left(\hat{\theta}_{s i}, \sigma_{s i}\right) \mathbf{U}\right\|^{2}} \quad i=1, \cdots, K,
$$

where $\mathbf{b}\left(\hat{\theta}_{s i}, \sigma_{s i}\right)=\left[\mathbf{b}_{1}\left(\hat{\theta}_{s i}, \sigma_{s i}\right), \mathbf{b}_{2}\left(\hat{\theta}_{s i}, \sigma_{s i}\right)\right]^{T}$ and $\hat{\theta}_{s i} \in \hat{\boldsymbol{\theta}}_{\mathrm{sec}}$.

By $1 \mathrm{D}$ search via $\sigma_{s i}$, we can obtain the second estimation of the angular spreads $\hat{\boldsymbol{\sigma}}_{\mathrm{sec}}=\left[\hat{\sigma}_{s 1}, \hat{\sigma}_{s 2}, \cdots, \hat{\sigma}_{s K}\right]$. 


\section{Simulation results}

In this section, we provide several simulation results to demonstrate the estimation performance of the proposed algorithm. And we exploit the root mean squared error as the figure of merit, which is expressed as

$$
R M S E=\sqrt{\frac{1}{N K} \sum_{n=1}^{N} \sum_{i=1}^{K}\left(a_{i}-\hat{a}_{i, n}\right)^{2}},
$$

where $N$ is the number of Monte-Carlo runs, $\hat{a}_{i, n}$ is the estimate of the $k$ th nominal DOA or angular spread of the $n$th Monte-Carlo run and $a_{i}$ is the perfect value. The signal-to-noise ratio (SNR) is defined as $S N R=\rho_{i} / \sigma_{n}^{2}$, where $\rho_{i}=E\left\{\left|v_{i}(t)\right|^{2}\right\}$ is the signal power of the $i$ th CD source.

Unless specifically explained, in all simulations, we set the number of snapshot $L$ as 500 . The times of Monte-Carlo run is 500 on average. The search range of nominal DOAs and angular spreads is $\left(0^{\circ}, 60^{\circ}\right)$ and $\left(0^{\circ}, 10^{\circ}\right)$, respectively. And we consider two CD sources of Gaussian distribution with nominal DOAs $\theta_{1}=10^{\circ}, \theta_{2}=30^{\circ}$ and angular spreads $\sigma_{1}=0.5^{\circ}, \sigma_{2}=2^{\circ}$ impinging on the array. The array is composed of two ULAs, where each subarray consist of $M=10$ sensors with half-wavelength inter-element spacing. The displacement $\delta$ between two subarrays is set as $\lambda / 10$.

In the first example, the estimation performance comparisons between the proposed algorithm, the ESPRIT-CD [8] and OPM-CD [11] algorithm are presented in Fig.2 (a) and Fig.2 (b). It could be clearly seen that the S-PM algorithm has better performance than ESPRIT-CD [8]. Meanwhile, the proposed algorithm can obtain close accuracy to the OPM-CD [11], which requires 2-D search.

In the second example, the RMSEs of the proposed algorithm versus the number of snapshots are plotted in Fig.3 (a) and Fig.3 (b). With $L$ increasing, the estimation performance of the S-PM algorithm are getting more precise.

In the third example, we present the RMSEs of the proposed algorithm versus the number of sensors in Fig.4 (a) and Fig.4 (b). It is illustrated that the parameter estimation performance of proposed algorithm is improved with $M$ increasing due to the diversity gain.

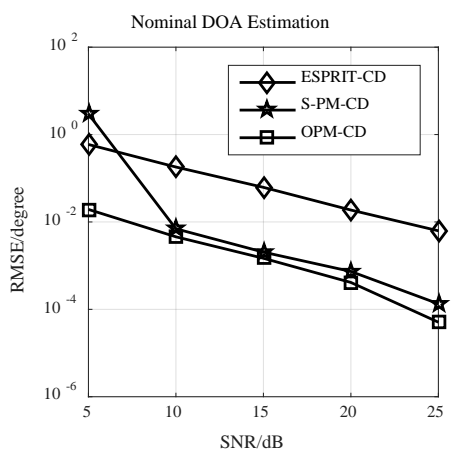

(a)

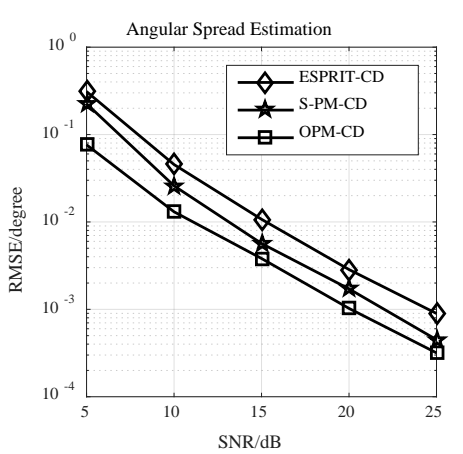

(b)

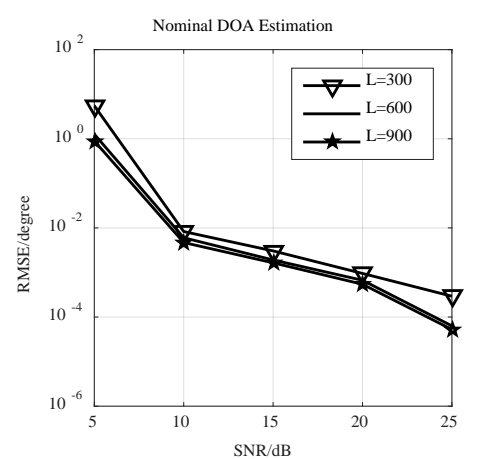

(a)

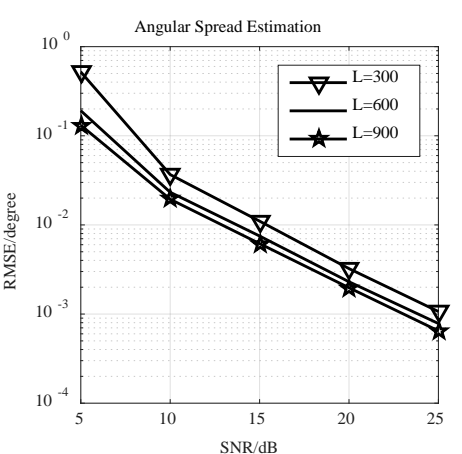

(b)
Fig.2 RMSEs versus SNR of the proposed algorithm, ESPRIT-CD [8] and OPM-CD [11]
Fig.3 RMSEs of the proposed algorithm versus different number of snapshots 

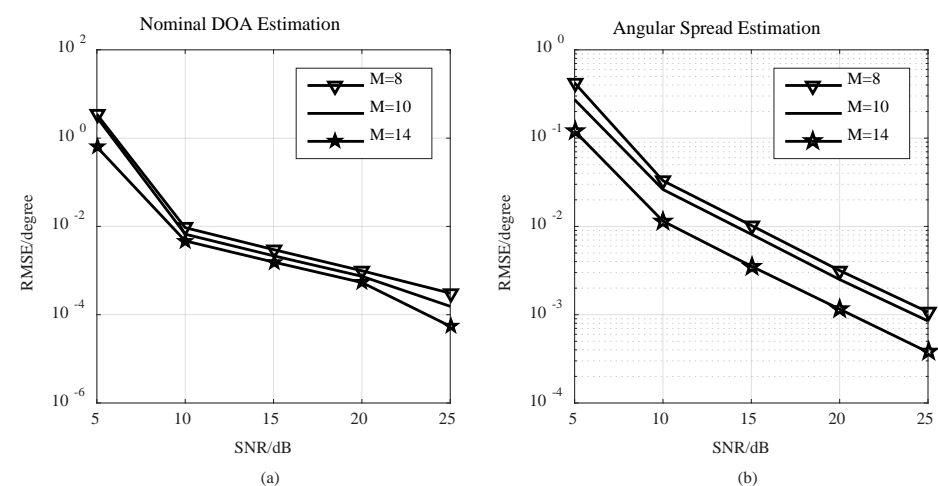

Fig.4 RMSEs of the proposed algorithm versus different number of sensors

\section{Summary}

In this letter, we proposed a successive PM algorithm for angular parameter estimation of CD source. Our algorithm based on propagator needs no 2-D spectral peak search and eigen-decomposition. So it has an advantage of lower complexity when compared to the OPM-CD [11] algorithm. And, in terms of estimation accuracy, the proposed algorithm outperforms the ESPRIT-CD [8] obviously.

\section{Acknowledgement}

This work is supported by China NSF Grants (61371169, 61601167), the open research fund of National Mobile Communications Research Laboratory, Southeast University (No.2015D030), Jiangsu NSF (BK20161489), the open research fund of State Key Laboratory of Millimeter Waves, Southeast University (No. K201826), and the Fundamental Research Funds for the Central Universities (NO: NE2017103).

\section{References}

[1] Zetterberg P, Ottersten B. The spectrum efficiency of a base station antenna array system for spatially selective transmission[J]. IEEE Transactions on Vehicular Technology (1995), 44(3):651-660.

[2] Asakura H, Matsumoto T. Cooperative signal reception and down-link beam forming in cellular mobile communications[J]. IEEE Transactions on Vehicular Technology (1999), 48(2):333-341.

[3] Asztely D, Ottersten B, Swindlehurst A L. Generalised array manifold model for wireless communication channels with local scattering[J]. IEE Proceedings - Radar, Sonar and Navigation, (1998), 145(1):51-57.

[4] Monakov A A. Observation of extended targets with antenna arrays[J]. IEEE Transactions on Aerospace \& Electronic Systems (2000), 36(1):297-302.

[5] Meng Y, Stoica P, Wong K M. Estimation of direction of arrival of spatially dispersed signals in array processing[J]. IEE Proceedings - Radar Sonar and Navigation (1996), 143(1):1-9.

[6] Trump T, Ottersten B. Estimation of nominal direction of arrival and angular spread using an array of sensors $\hat{z}[\mathrm{~J}]$. Signal Processing (1996), 50(1-2):57-69.

[7] Valaee S, Champagne B, Kabal P. Parametric localization of distributed sources[J]. IEEE Transactions on Signal Processing (1995), 43(9):2144-2153.

[8] Shahbazpanahi S, Valaee S, Bastani M H. Distributed source localization using ESPRIT algorithm[J]. IEEE Transactions on Signal Processing (2001), 49(10):2169-2178.

[9] S. Marcos, A. Marsal, and M. Benidir, "The propagator method for source bearing estimation," Signal Processing, vol. 42, no. 2, pp. 121-138 (1995).

[10]N. Tayem and H. M. Kwon, "L-shape 2-dimensional arrival angle estimation with propagator method,” IEEE Transactions on Antennas and Propagation, vol. 53, no. 5, pp. 1622-1630, May 
(2005).

[11]Han Yinghua. Research on distributed source parameter estimation algorithm in local scattering environment [D]. Northeastern University (2008).

[12]Li J, Zhang X, Chen H. Improved two-dimensional DOA estimation algorithm for two-parallel uniform linear arrays using propagator method[J]. Signal Processing (2012), 92(12):3032-3038.

[13]R. Schmidt, "Multiple emitter location and signal parameter estimation,” IEEE Trans. Antennas Propagotor, vol. 34, no. 3, pp. 276-280, Mar (1986).

[14]S. Marcos, A. Marsal, and M. Benidir, "The propagator method for source bearing estimation," Signal Processing, vol. 42, no. 2, pp. 121-138 (1995). 